\title{
Basic Understanding and Perspectives for Coronavirus
}

\author{
Rui-L* \\ School of Pharmacy, Xi'an Jiaotong University, Xi'an, PR China
}

*Corresponding author: Rui-L, School of Pharmacy, Xi'an Jiaotong University, Xi'an, PR China, Email: Irlxjtu1987@xjtu.edu.cn

\section{Perspective}

Volume 5 Issue 1

Received Date: March 03, 2020

Published Date: March 18, 2020

DOI: $10.23880 /$ nnoa- 16000179

\section{Perspective}

Recently, a novel coronavirus suddenly appeared in China and around the world, and it caused a terrible outbreak of pneumonia, which poses a great threat and challenge to global public health. Although the tremendous efforts in public healthcare have been made, conquering the viruses-caused infectious diseases remains a huge challenging and endless task. Coronaviruses belong to the family of Coronaviridae and may cause respiratory and neurological diseases [1]. So far, six human coronaviruses (HCoVs) have been identified such as the HCoV-229E, HCoV-HKU1, HCoV-OC43, HCoVNL63, severe acute respiratory syndrome coronavirus (SARS$\mathrm{CoV}$ ), and Middle East respiratory syndrome coronavirus (MERSCoV) [2]. Lately, the novel coronavirus (SARS-CoV-2) emerged in China and then it rapidly spread almost all the world in a very short time. On 11 February 2020, directorgeneral of the World Health Organization (WHO) announced that the pneumonia infected by the new coronavirus was named "COVID-19" (Corona Virus Disease 2019). It has also caused a enormous challenging and concern for the global public health community. As of 2 March 2020, there were 89,081 confirmed cases with COVID-19 and 3,057 deaths. At present, the diagnosis of COVID-19 mainly relies on the detection of the coronavirus RNA [2]. The determinations of SARS-CoV-2 RNA were considered as an effective tool for the early diagnosis of COVID-19 [2]. Therefore, exploring a rapid and accurate detection of COVID-19 is very important for promoting efficient control of the virus and caring the people's health. Nucleic acid detection methods have been fleetly developed and it has become a revolutionary technology for virus detection. Note that the polymerase chain reaction (PCR)-based analysis method as the "gold standard" has been used for virus detection because it has the good analytical performances such as the rapid detection, high sensitivity and specificity [3]. To improve both the specificity and sensitivity of the analysis method, researchers explored some novel PCRbased methods such as real-time reverse transcriptase-PCR (RT-PCR) and multiplex real-time RT-PCR for the detection of
COVID-19 RNA [2]. But beyond that, the isothermal nucleic acid amplification and microarray-based methods were also developed for the detection of coronavirus RNA. However, each of the analysis methods mentioned above has its own advantages and inevitable disadvantages. Generally, the PCR is used for virus identification, but its analysis needs various instruments and well-educated analysts [2]. With regard to the microarray-based method for the detection of coronavirus, one of the biggest drawbacks is the high cost that further hinders its large-scale application, especially in poor and remote areas. Thus, it is an urgent need to develop more effective and practical strategies to surmount the flaws of existing methods in future. I personally think that the developing highly sensitive fluorescence or colorimetric analysis methods based on nanotechnology for the analysis of the coronavirus would be possible in the future. We may take full advantage of nanotechnology to accurately monitor the COVID-19 later, instead of the conventional PCR diagnostic methods. From a therapeutic point of view, inspired by the research approaches of current nanomedicine, we might as well boldly imagine and explore the treatments of the COVID-19 by using some safe nanomaterials. Overall, I believe that with the joint efforts of people around the world and scientific prevention and control, we will certainly defeat the "COVID-19" soon.

\section{References}

1. Zhang N, Wang L, Deng X, Liang R, Su M, et al. (2020) Recent advances in the detection of respiratory virus infection in humans. Journal of Medical Virology 92(4): 408-417.

2. Shen M, Zhou Y, Ye J, Abdu Ahmed Abdullah AL, Kang Y, Zeng S, et al. (2020) Recent advances and perspectives of nucleic acid detection for coronavirus, Journal of Pharmaceutical Analysis. 
3. Uhlenhaut C, Cohen JI, Pavletic S, Gabor I, Juan Carlos GB, et al. (2012) Use of a novel virus detection assay to identify coronavirus HKU1 in the lungs of a hematopoietic stem cell transplant recipient with fatal pneumonia. Transplant Infectious Disease 14(1): 79-85.

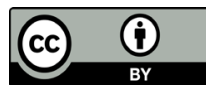

